\title{
Intracellular uptake of and sensing with SERS-active hybrid exosomes: insight into a role of metal nanoparticles
}

\author{
Hui Chen' (iD), Caixia Luo', Moyu Yang ${ }^{1}$, Junying Li' ${ }^{1}$, Pei Ma*,1 \& Xuedian Zhang1 \\ ${ }^{1}$ Shanghai Key Laboratory of Contemporary Optics System, School of Optical-Electrical \& Computer Engineering, University of \\ Shanghai for Science \& Technology, 200093, Shanghai, PR China \\ *Author for correspondence: peima@usst.edu.cn
}

\begin{abstract}
Aim: Exosomes, known as novel biocompatible vesicles, have attracted much interest. This makes it urgent to observe exosomes at the visually cellular or subcellular levels. Methods: Herein, we constructed a new kind of exosome/metal nanohybrid and employed a surface-enhanced Raman scattering technique to study the intracellular behaviors of hybrid exosomes. Results: Experimental results revealed that hybrid exosomes were internalized mainly through clathrin-mediated endocytosis and thereafter transported to lysosomes. The metal nanoparticles in the hybrid were demonstrated to have little effect on exosomal characteristics while serving as surface-enhanced Raman scattering generators. Conclusion: This study is significant for removing the barrier in designing programmable exosome/metal nanohybrids, which will greatly improve the utility of exosomal nanohybrids for therapeutics, such as multifunctional drug-delivery systems.
\end{abstract}

First draft submitted: 13 November 2019; Accepted for publication: 10 January 2020; Published online: 27 March 2020

Keywords: exosomes $\bullet$ intracellular sensing $\bullet$ metal nanoparticles $\bullet$ nanohybrids $\bullet$ surface-enhanced Raman scattering

Exosomes are nanoscale extracellular vesicles that can be secreted by numerous cell types. The exosome vesicle is enclosed by a phospholipid bilayer with a size ranging from 30 to $150 \mathrm{~nm}$ [1]. Based on the trafficking process, exosomes have been recognized to play an integral role in the communication between cells [2]. By interacting with the cells, exosomes are able to efficiently deliver a variety of the parent cell-related bioactive molecules (i.e., microRNAs) to recipient cells [3], which consequently regulate the behavior of recipient cells and influence the involved physiological or pathological processes $[4,5]$. This has led to the burgeoning research of using exosomes as diagnostic biomarkers [6,7] and therapeutic targets [8]. Recently, exosomes are of special interest for being used as drug carriers due to their full biocompatibility $[9,10]$.

Although a variety of nanoparticles, such as liposomes and metal nanoparticles, have been extensively studied for drug delivery, clinical applications are still limited [11]. In contrast, exosomes have potential strengths of noncytotoxicity and nonimmunogenicity [12]. Deep understanding of the interaction between exosomes and cells is always a crucial prerequisite for efficiently exploiting exosomes as vectors for drug delivery. Up to now, a number of methods for the labeling and sensing of exosomes have been proposed.

The most widely used approach is to label purified exosomes with different fluorescent probes and then identify the intracellular exosomes by using flow cytometry or fluorescence microscopy. Fluorescent probes, such as lipophilic dyes (PKH67, DiR) [13,14], green fluorescent protein [15] and quantum dots (carbon quantum dots, silicon quantum dots) $[16,17]$ have been utilized for exosome imaging and are proved to offer good feasibility for exosome sensing. In addition, iron oxide nanoparticles could be embedded inside exosomes for MRI, which was constructed by culturing their parent cells with iron oxide nanoparticles and the nanoparticles were subsequently incorporated into the secreted exosomes [18]. Exosomes can be labeled with gold nanoparticles for computed tomography (CT) imaging [19] using a mouse model of focal brain ischemia, which can serve as a diagnostic tool for brain disorders. When being radiolabeled with $(99 \mathrm{~m}) \mathrm{Tc}-\mathrm{HMPAO}$, single-photon emission computed tomography (SPECT)/CT

Future Medicine 


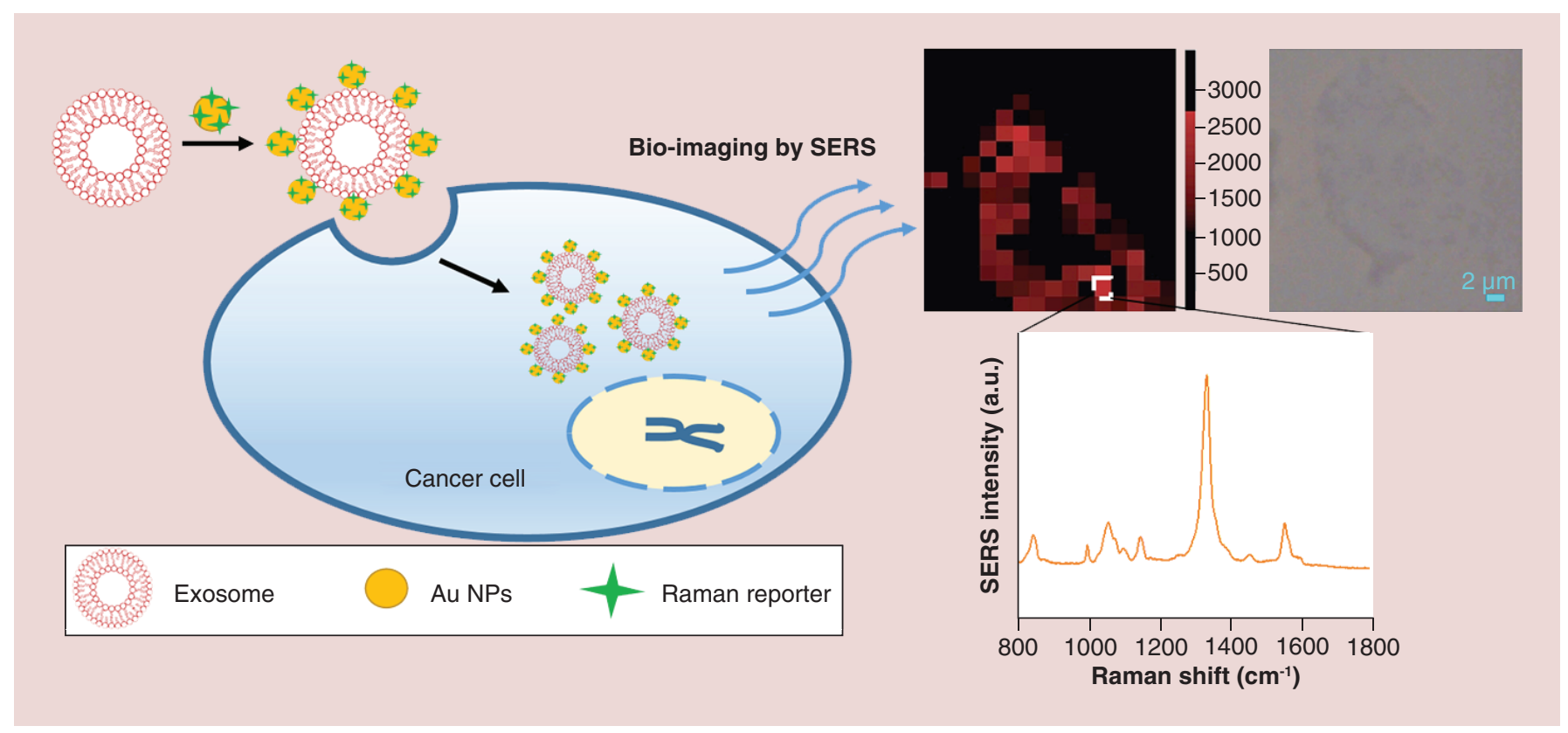

Figure 1. Sketch for intracellular tracking of the surface-enhanced Raman scattering-active exosomes. Au NP: Gold nanoparticle; SERS: Surface-enhanced Raman scattering.

imaging can be used to track the distribution of exosomes in vivo, which exhibits that exosomes were more efficiently accumulated in the liver [20]. However, fluorescence imaging may sometimes encounter strong photobleaching or spectral overlapping [21] and MRI may suffer from long acquisition times and poor sensitivity. Although CT and SPECT/CT have provided good feasibility for exosome imaging in vivo, they are confronted with poor sensitivity and an increasing radiation burden especially in repeated scans, which limit multiplexing. In addition, CT and SPECT/CT usually display a spatial resolution of several tens of microns which can be applied to trace exosomes in tissues or organs. However, this spatial resolution is obviously inadequate for the study of cellular or subcellular behaviors because eukaryotic cells are typically several microns to several tens of microns in size. To observe exosomes at the visually cellular or subcellular levels, an imaging technique with higher resolution is desired. Due to the abundant fingerprint spectra with highly distinguishable and sensitive properties, the surface-enhanced Raman scattering (SERS) technology presents superior spatial resolution and exciting ability of multiplexed sensing of biological processes inside living cells [22]. By using plasmonic structures, SERS can boost the Raman signals by a factor of up to $10^{14}$ [23,24]. Giving that metal nanoparticles are usually employed as enhancing substrates, it is possible to achieve the transition of an exosome to a SERS traceable exosome by incorporating metal nanoparticles with it.

It is also notable that investigations of the exosomes labeled with quantum dots or iron oxide nanoparticles in the previous works rarely refer to the influence of conjugated nanoparticles. However, understanding whether the nanoparticles affect the functionality of exosomes is extremely important for their better utility in intracellular signaling and sensing.

Taking all the abovementioned into account, herein, we developed a SERS-active exosome and monitored the intracellular trafficking processes of exosomes through a SERS technique. A simple and clear principle illustration was demonstrated in Figure 1. To prepare such a SERS-active exosome, Au nanoparticles modified with Raman reporter groups were conjugated to an exosome. Au nanoparticles served as SERS-active generators, while the exosome was used as a vehicle for loading and delivery of Au nanoparticles into the cells. We thereafter investigated the potential pathways involved in the internalization of exosomes using the well-characterized endocytic HeLa cell model. This was performed by monitoring the SERS signals of exosomes within the cells pretreated with several endocytic inhibitors, which can selectively block the specific uptake pathways. We demonstrate that the SERS technique facilitates the sensing of cellular uptake of hybrid exosomes and visualizes the cellular uptake results. The intracellular fate of hybrid exosomes after the internalization as well as the influence of metal nanoparticles on exosomal properties were also investigated. This work provides a valuable insight into the role of metal 
nanoparticles in hybrid exosomes, which serves as a crucial precondition for the development and application of exosomal nanohybrids in therapeutics.

\section{Materials \& methods}

\section{Materials}

Hydrogen tetrachloroaurate (III) trihydrate $\left(\mathrm{HAuCl}_{4} \cdot 3 \mathrm{H}_{2} \mathrm{O}\right)$, 5,5'-dithiobis-(2-nitrobenzoic acid) (DTNB), poly(allylamine hydrochloride) (PAH; MW 17,500) were purchased from Sigma-Aldrich (MO, USA). Trisodium citrate dehydrate and absolute ethanol were purchased from Sinopharm Chemical Reagent Co., Ltd (Shanghai, China). Phosphate-buffered saline (PBS; 10 mM, pH 7.4) was purchased from Nanjing KeyGen Biotechnology Co., Ltd (Nanjing, China). Chlorpromazine, nystatin and amiloride hydrochloride were purchased from Shanghai Macklin Biochemical Co., Ltd (Shanghai, China). ExoQuick-TC Exosome Precipitation Solution was purchased from System Biosciences (CA, USA). In all of the experiments, deionized water with a resistivity of $18.2 \mathrm{M} \Omega \mathrm{cm}^{-1}$ was used.

\section{Cell culture}

HeLa cells of human cervical cancer were purchased from China Type Culture Collection (Shanghai, China). The cells were cultured in minimum essential medium (MEM) medium supplemented with $10 \%$ fetal bovine serum as well as $1 \%$ penicillin-streptomycin. The cell culture was performed under a standard condition $\left(5 \% \mathrm{CO}_{2}, 37^{\circ} \mathrm{C}\right)$.

\section{Purification \& characterization of exosomes}

To isolate exosomes, HeLa cells were seeded onto a culture flask (Corning, MA, USA). When the cells were cultured to $70-80 \%$ confluence, the culture medium was collected for isolation. Ten milliliters of the medium was first centrifuged at $10,000 \times g$ for $15 \mathrm{~min}$. After removing the cell debris, the obtained supernatant was added by $2 \mathrm{ml}$ of exosome precipitation solution (ExoQuick-TC) and kept at $4^{\circ} \mathrm{C}$ overnight. The mixed solution was then centrifuged at $1500 \times g$ for $30 \mathrm{~min}$. After removing the fluid, the centrifugation at $1500 \times g$ for $5 \mathrm{~min}$ was conducted once more to further remove the remaining fluid. Finally, the exosome pellet was suspended in $200 \mu \mathrm{l}$ of PBS and stored at $-20^{\circ} \mathrm{C}$. For transmission electron microscopy (TEM) imaging, isolated exosomes were placed onto the carbon coated grids for $15 \mathrm{~min}$. Then, excess liquid was carefully absorbed away from the edge of the copper mesh through the filter paper. Exosomes on the copper mesh were then stained for contrast by $2 \%$ phosphotungstic acid for $10 \mathrm{~min}$. Finally, the specimen was subject to TEM observation.

\section{Preparation of SERS-active exosomes}

Au nanoparticles were synthesized using the sodium citrate reduction method. Typically, $\operatorname{HAuCl}_{4}(2 \mathrm{ml}, 1 \% \mathrm{w} / \mathrm{w})$ was added to $200 \mathrm{ml}$ of deionized water and the mixture was stirred and heated to boiling. Trisodium citrate $(8 \mathrm{ml}$, $1 \% \mathrm{w} / \mathrm{w}$ ) was then added and the mixed solution was boiled and stirred continuously until it began to show a wine red. After that, the solution was kept boiling for $15 \mathrm{~min}$ and cooled down to room temperature. Au nanoparticles were labeled with Raman reporters by incubating $5 \mathrm{ml}$ of Au colloidal solution with DTNB $\left(10 \mu \mathrm{l}, 1 \times 10^{-2} \mathrm{M}\right)$ for $2 \mathrm{~h}$. Then, DTNB-labeled Au nanoparticles were separated from the solution by centrifugation at $6500 \mathrm{rpm}$ for $40 \mathrm{~min}$. After the removal of excess DTNB, the sediment was suspended in $1 \mathrm{ml}$ of deionized water. Under gentle stirring, PAH $\left(250 \mu \mathrm{l}, 10 \mathrm{mg} \mathrm{ml}^{-1}\right.$, in $\left.1 \times 10^{-2} \mathrm{M} \mathrm{NaCl}\right)$ was mixed with the DTNB-labelled Au nanoparticles. The mixture was reacted for $1 \mathrm{~h}$ to modify the surfaces of Au nanoparticles with amino groups. At last, the mixture was centrifuged at $6500 \mathrm{rpm}$ for $30 \mathrm{~min}$ to remove the excess PAH. The sediment was resuspended in $1 \mathrm{ml}$ of water.

SERS-active exosomes were obtained through the interaction of glutaraldehyde and amino. Typically, $500 \mu \mathrm{l}$ of PAH modified Au@DTNB was incubated with $10 \mu \mathrm{l}$ of exosomes under mild shaking. Then, $10 \mu \mathrm{l}$ of glutaraldehyde (50\%) was added and the mixture was continuously shaken for $2 \mathrm{~h}$. The mixed solution was then centrifuged at $1500 \times \mathrm{g}$ for $20 \mathrm{~min}$. The obtained hybrid exosomes were suspended in $50 \mu \mathrm{lof}$ PBS ( $\mathrm{pH} 7.4$ ) and stored at -20 or $-80^{\circ} \mathrm{C}$.

\section{Influence of hybrid exosomes on cell proliferation}

The cytotoxicity of pure exosomes and hybrid exosomes against HeLa cells were evaluated by 3-(4,5-dimethylthiazol2-yl)-2,5-diphenyltetrazolium bromide (MTT) assay. Briefly, HeLa cells were seeded onto 96-well plates $(100 \mu \mathrm{l} /$ well) and incubated for $24 \mathrm{~h}$. Then, the original medium was replaced by the fresh culture medium 
containing exosomes or hybrid exosomes with final concentrations at 0, 20, 40, 40 and $80 \mu \mathrm{g} \mathrm{ml}^{-1}$. Cells incubated only with culture medium were used as a control. After 12, 24 and 48 h, $50 \mu$ of MTT solution (MTT buffer to dilution buffer 1:4) was added to each well. After $4 \mathrm{~h}$, the medium in each well was removed and $150 \mu \mathrm{l}$ of DMSO was added. The absorbance of the solution in each well at $490 \mathrm{~nm}$ was measured to calculate the percentage of viable cells.

\section{Cellular uptake \& intracellular fate studies of hybrid exosomes}

As a well characterized endocytic cell model, HeLa cells were chosen to investigate the uptake mechanism of hybrid exosomes. HeLa cells were first seeded onto culture dish and cultured overnight. Hybrid exosomes were then added to the culture dish (the volume ratio of hybrid exosomes to culture media was 1:5). After different time durations, the medium was removed and the culture dish was washed with PBS three-times before SERS observation.

To monitor the cellular uptake processes of hybrid exosomes, HeLa cells were pretreated with the inhibitors for $2 \mathrm{~h}$. Then, hybrid exosomes were added and the culture was prolonged for $4 \mathrm{~h}$ at $37^{\circ} \mathrm{C}$. After that, the old medium containing inhibitors and hybrid exosomes were removed. The cells were washed with PBS three-times and subject to SERS acquisition. Specifically, to inhibit clathrin-mediated endocytosis, cells were pretreated with chlorpromazine $\left(10 \mu \mathrm{g} \mathrm{ml}^{-1}\right)$. Caveolae-mediated endocytosis was inhibited by pretreating the cells with nystatin $\left(50 \mu \mathrm{g} \mathrm{ml}^{-1}\right)$ and macropinocytosis was inhibited with amiloride $\left(10 \mu \mathrm{g} \mathrm{ml}^{-1}\right)$. HeLa cells pre-incubated at $4^{\circ} \mathrm{C}$ for $2 \mathrm{~h}$ were used as a negative control to investigate the energy-dependent cellular uptake. HeLa cells without inhibitor treatment were used as a positive control. After removing the old medium, the cells were also washed with PBS and then observed with the confocal Raman spectroscopy. All experiments were conducted in serum-free medium.

For the investigation of intracellular fate, the cells were incubated with hybrid exosomes for different time durations and then treated with Lyso-tracker to locate the acidic lysosomes before observation. For comparison experiments, the cells were incubated with lipophilic dye chloromethylbenzamido dialkylcarbocyanine (CM-DiI)labeled exosomes or gold nanoprobes under the same conditions. After the observation under a confocal microscope, the colocalization of exosomes with lysosomes was analyzed.

\section{Instruments \& measurements}

$\zeta$ potential was measured using a Malvern Zetasizer Nano ZS 90. Extinction spectra were measured by a Shimadzu UV-3600 PC spectrophotometer with quartz cuvettes of $1 \mathrm{~cm}$ path length. TEM images were obtained with a JEM-2100F, JEOL electron microscope. SERS measurements of the cells were performed with the confocal Raman spectroscopy (Xplora, Horiba) at $638 \mathrm{~nm}$ excitation. The scattering light was collected by a water immersion $60 \times$ objective lens $(\mathrm{NA}=1)$ to the charge coupled device (CCD). SERS spectra were acquired with the accumulation time of 6 s. SERS mapping was also performed by the confocal Raman spectroscopy with a $60 \times$ objective lens at a step size of $2 \mu \mathrm{m}$ and the accumulation time for each point was $6 \mathrm{~s}$. Each pixel in the mapping images was created through the Raman intensity of $1333 \mathrm{~cm}^{-1}$ band. A serials of SERS spectra for all mapping pixels in each image were recorded to obtain an average SERS spectrum. This was performed for ten different cell mapping images. Then, ten acquired average SERS spectra were averaged again to get a final average spectrum. The averaged signal intensity and standard deviation were also calculated from ten cell mapping images.

For intracellular fate, SERS/fluorescence images were observed by a confocal microscope (Andor Dragonfly 200) with an inverted oil immersion $60 \times$ objective lens $(\mathrm{NA}=1.4)$. The excitation at $637 \mathrm{~nm}$ was used for SERS observation and the photons of 685-715 nm were collected for SERS imaging of hybrid exosomes. While for the imaging of lysosomes, the excitation wavelength was $488 \mathrm{~nm}$ and the photons collected from 505 to $535 \mathrm{~nm}$ were used for fluorescence imaging. For the imaging of CM-DiI-labeled exosomes, the excitation at $543 \mathrm{~nm}$ was used and the emission light from 585 to $615 \mathrm{~nm}$ was collected. By randomly choosing ten cells, the mean signal intensity per cell was obtained. The colocalization ratio of red and green signals in the merged images was quantitatively analyzed by the Pearson's coefficient and overlap coefficient. This was conducted on ten randomly selected regions to obtain an average correlation coefficient.

\section{Results}

Characterization of SERS-active exosomes

The preparation of SERS-active hybrid exosomes was constructed in three steps. First, exosomes were isolated from the conditioned cell culture medium using an exosome precipitation kit under standard protocols. The obtained exosomes showed a negative $\zeta$ potential of $-20.7 \mathrm{mV}$ (Figure 2A). TEM images were shown in Figure 2B-D to 

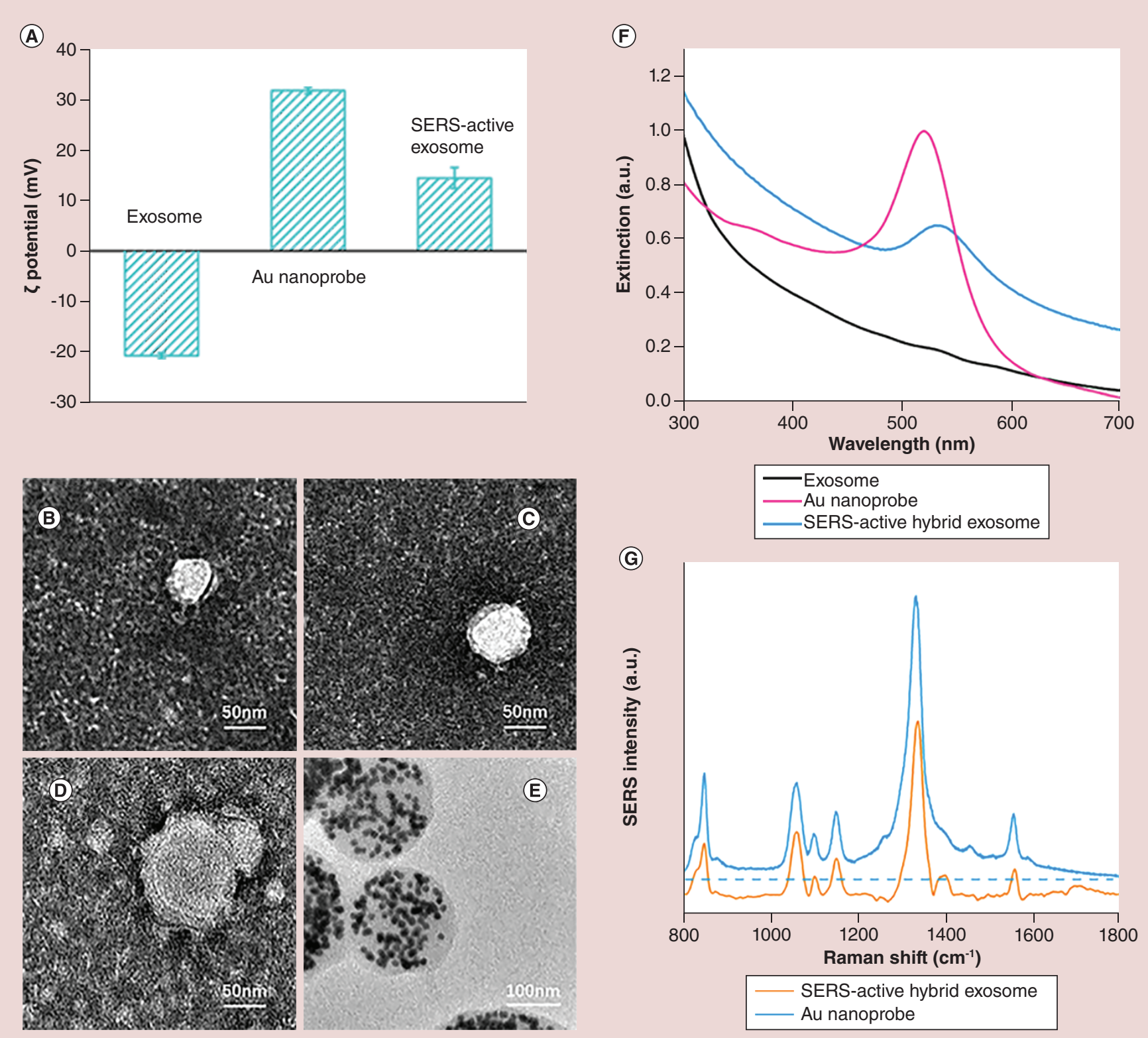

Figure 2. Characterization of hybrid exosomes. (A) $\zeta$ potential of exosomes, Au nanoprobes and SERS-active hybrid exosomes measured with dynamic light scattering. Transmission electron microscopy images (B-D) and SERS-active hybrid exosomes (E). (F) Extinction spectra of the exosomes, Au nanoprobes and SERS-active hybrid exosomes. (G) SERS spectra of the nanoprobes and SERS-active hybrid exosomes. The spectra were placed in parallel for clarity.

SERS: Surface-enhanced Raman scattering.

confirm the successful exosome isolation. Three typical exosomes with different sizes were presented. The diameters of the exosomes in Figure 2B-D were 60, 80 and $110 \mathrm{~nm}$, respectively. In the second step, Au nanoparticles were synthesized according to the previous literature [25]. As shown in Supplementary Figure 1, gold nanoparticles were observed by TEM to be uniform and monodispersed in the diameter of $15.3 \mathrm{~nm}$. The extinction spectrum of Au nanoparticles exhibited a single surface plasmon resonance (SPR) band at $519 \mathrm{~nm}$ (Supplementary Figure 2). Au nanoparticles were then tagged with DTNB molecules and followed by the modification of PAH polymer. The DTNB tagged and PAH-coated Au nanoparticles were termed as Au nanoprobes which exhibited a positive surface charge of $+31.7 \mathrm{mV}$ (Figure $2 \mathrm{~A}$ ). The SPR band of the Au nanoprobes slightly redshifted to $524 \mathrm{~nm}$ (Supplementary Figure 2), which was due to the increase of refractive index around Au nanoparticles caused by 
the DTNB and PAH molecules [26]. Finally, the exosome was labeled with Au nanoprobes using glutaraldehyde as bridging linkers since amino groups were presented on both of the exosomes [27] and Au nanoprobes. Upon the loading of Au nanoprobes, SERS-active hybrid exosomes exhibited a $\zeta$ potential of $+14.5 \mathrm{mV}$ (Figure 2A). Compared to exosomes, the surface charge shifted from -20.7 to $+14.5 \mathrm{mV}$, which confirmed the conjugation of positively charged Au nanoprobes.

Further, to visually verify the conjugation of Au nanoprobes with exosome, the obtained hybrid exosomes were characterized by TEM and UV-Vis spectroscopy. Compared with pure exosomes, hybrid exosomes exhibited vesicles loading with Au nanoparticles (Figure 2E). The size of exosomes in Figure 2E was observed to be $200 \mathrm{~nm}$ (out range of 30 to $150 \mathrm{~nm}$ ), which may be attributed to the size increase at low temperature storage [28]. Hybrid exosomes presented an obvious SPR band at $536 \mathrm{~nm}$ in the extinction spectrum (Figure 2F). Compared with Au nanoprobes, the SPR band of hybrid exosomes exhibited a redshift. This may be ascribed to the slightly aggregated gold nanoprobes in the hybrids [29]. Compared with exosomes, the distinctly changed extinction spectrum of hybrid exosomes was caused by the conjugation of Au nanoprobes. All the above results confirmed the successful transition of exosomes to SERS-active hybrid exosomes.

\section{SERS performance of SERS-active exosomes}

DTNB molecules were immobilized on the surfaces of gold nanoparticles through $\mathrm{Au}-\mathrm{S}$ bonds to generate $\mathrm{Au}$ nanoprobes. As shown in Figure 2G, Au nanoprobes exhibited characteristic SERS bands of DTNB molecules. Briefly, SERS bands at $1067,1152,1333$ and $1558 \mathrm{~cm}^{-1}$ were assigned to the succinimidyl N-C-O stretch overlapping with aromatic ring modes, the $\mathrm{C}-\mathrm{H}$ deformation modes, the symmetric stretch of the nitro groups and the aromatic ring $\mathrm{C}-\mathrm{C}$ stretching modes, respectively $[29,30]$.

Hybrid exosomes displayed a similar SERS spectrum to Au nanoprobes (Figure 2G). The distinct SERS properties of hybrid exosomes make them optical traceable in living cells. Before the cellular internalization, it is important to confirm that hybrid exosomes can maintain their structure and characteristics in biologic environments. The colloidal stability of hybrid exosomes in the culture medium was investigated (Supplementary Figure 3). After a long incubation period, the hydrodynamic size only displayed a slight increase, which was most likely originated from the absorption of proteins or amino acids in the culture medium. Despite this, hybrid exosomes generally exhibited excellent stability in the cell culture medium over $24 \mathrm{~h}$. Additionally, the influence of hybrid exosomes on cell proliferation was investigated since low toxicity is essential for the designed nanocarriers. As shown in Supplementary Figure 4, the viability of HeLa cells incubated with exosomes was always more than $92 \%$ which indicated that cytotoxicity of pure exosomes against HeLa cells was negligible. Hybrid exosomes showed a little higher cytotoxicity than pure exosomes, which was attributed to the conjugation of gold nanoparticles. As the incubation time prolonged or the concentration of hybrid exosomes increased, the viability of HeLa cells was slightly decreased. However, even after $48 \mathrm{~h}$ incubation with hybrid exosomes at a high concentration of $80 \mu \mathrm{g} / \mathrm{ml}$, high viability of HeLa cells (82.51\%) was achieved.

After HeLa cells were incubated with SERS-active hybrid exosomes for $4 \mathrm{~h}$, intracellular hybrid exosomes were tracked by detecting the SERS signals (Figure 3A). The bright-field image (Figure 3B) was presented to locate the cell in Figure 3A. The SERS spectra of hybrid exosomes at different spots inside the cell in the merged image (Figure 3C) were shown in Figure 3D. Each spectrum detected from the individual spot undoubtedly described the SERS fingerprint of DTNB. Thus, hybrid exosomes are capable of maintaining the SERS properties inside the cells.

\section{Cellular uptake mechanism of exosomes}

SERS signals were employed to investigate the cellular uptake of hybrid exosomes. Generally, endocytosis is known as a pathway through which the cells internalize various extracellular materials [31]. The endocytosis can be classified into pinocytosis and phagocytosis [32]. Specifically, pinocytosis is involved in the uptake of small particles while phagocytosis is the pathway for large materials. For the presented nanohybrids, pinocytosis is involved and can be further classified into three distinct mechanisms, including clathrin-mediated endocytosis, caveolae-mediated endocytosis and macropinocytosis, corresponding to different participating proteins [33]. Previous studies have demonstrated that liposomes [33] and graphene oxide [34] entered the cells mainly through clathrin-mediated endocytosis. To elucidate the mechanism of cellular uptake of exosomes, HeLa cells were pretreated with several inhibitors to selectively block the specific pathways. Chlorpromazine [35], amiloride [36] and nystatin [37] were chosen as the inhibitors, which have been known to disturb the cellular entry pathways mediated by clathrin, actin and 

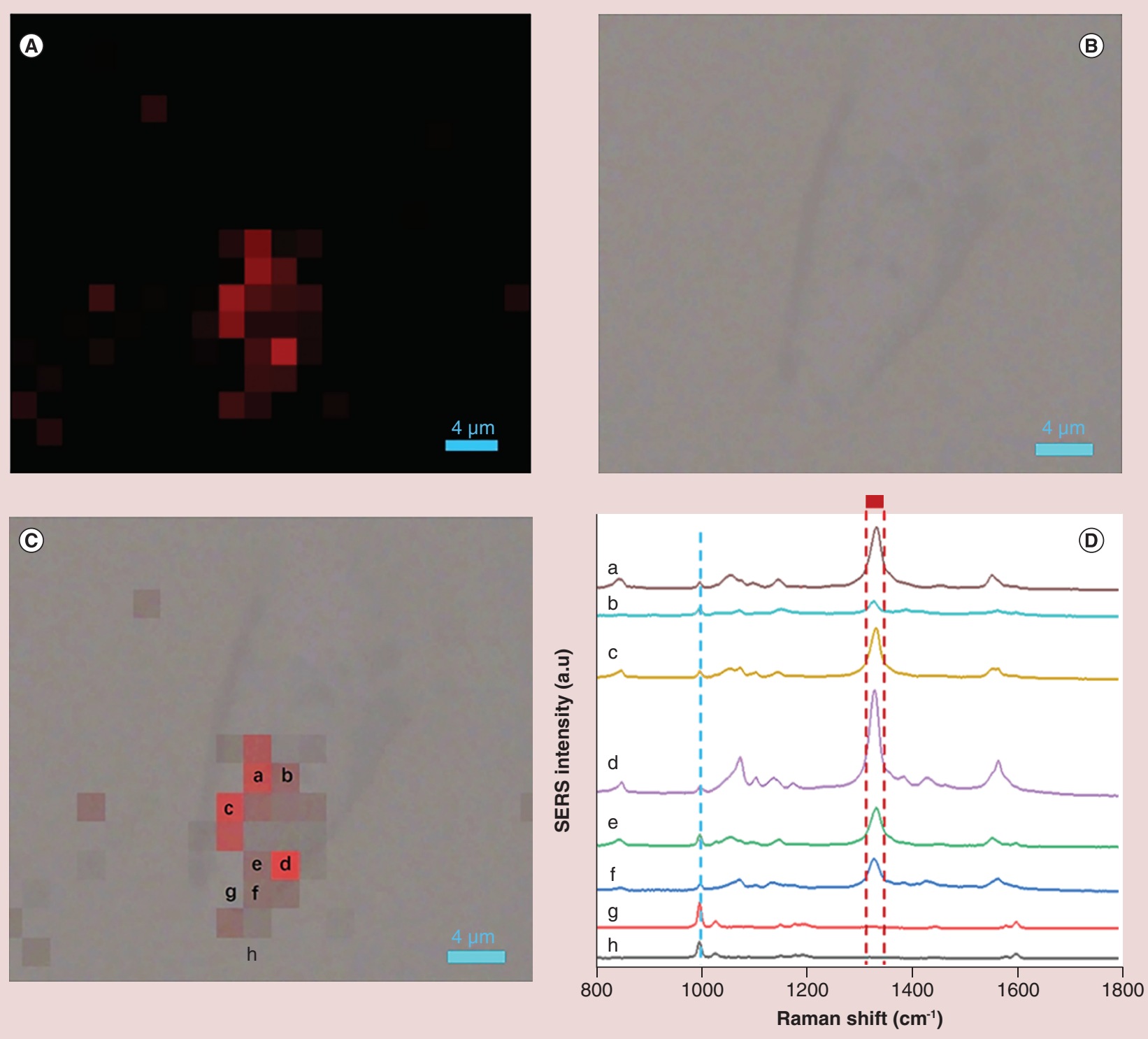

Figure 3. Intracellular imaging of hybrid exosomes using surface-enhanced Raman scattering. (A) SERS mapping and (B) bright-field and (C) merged images of the HeLa cell treated with SERS-active hybrid exosomes for $4 \mathrm{~h}$. (D) SERS spectra of different spots from the HeLa cell marked in (C). Each pixel represented the SERS intensity of $1333 \mathrm{~cm}^{-1}$ band. The band indicated by blue dashed line at $1001 \mathrm{~cm}^{-1}$ belongs to the culture dish.

SERS: Surface-enhanced Raman scattering.

caveolae, respectively. Additionally, HeLa cells without inhibitor treatment under the same condition were used as a positive control while cells pretreated at $4^{\circ} \mathrm{C}$ as a negative control.

After different treatments, the pretreated cells were followed by incubation with SERS-active hybrid exosomes for $4 \mathrm{~h}$. Afterward, the cells were washed with PBS and subject to SERS observation by the confocal Raman spectroscopy. For each experiment, ten measurements were conducted and the data was averaged. In the positive control experiment (Figure 4A \& B), the cells exhibited intense SERS activity which indicated the efficient intracellular uptake of SERS-active hybrid exosomes. In the negative control experiment at $4^{\circ} \mathrm{C}$, the cellular uptake efficiency was significantly decreased and SERS signals inside the cells were almost invisible (Figure 4C \& D). Since endocytosis is an energy-dependent process which can be inhibited under the low temperature, a conclusion was drawn that HeLa cells internalized hybrid exosomes through the energy-dependent endocytosis. Meanwhile, pretreatment with chlorpromazine resulted in a substantial decrease of SERS signals (Figure 4E \& F), which 

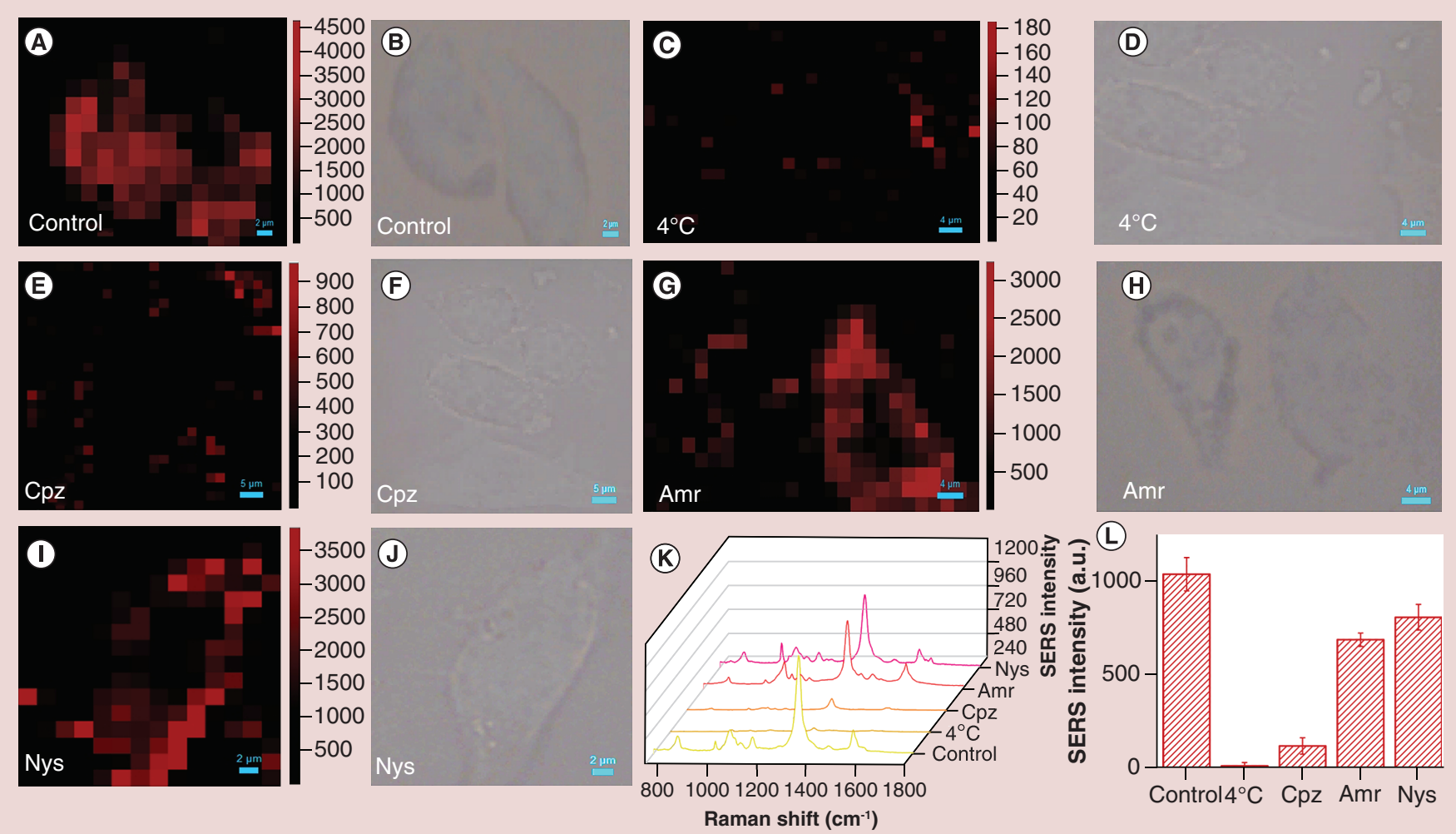

Figure 4. Uptake mechanism of hybrid exosomes. (A-J) Cellular uptake inhibition study for SERS-active hybrid exosomes. Three different inhibitors including clathrin inhibitor $\mathrm{Cpz}$, macropinocytosis inhibitor Amr and caveolae inhibitor Nys at different concentrations were used: $\mathrm{Cpz}\left(10 \mu \mathrm{g} \mathrm{ml}^{-1}\right)$; Amr $\left(10 \mu \mathrm{g} \mathrm{ml}^{-1}\right)$; Nys $\left(50 \mu \mathrm{g} \mathrm{ml}^{-1}\right)$. Cells without the inhibitor treatment were used as a positive control while cells pretreatment at $4^{\circ} \mathrm{C}$ were used to investigate the energy-dependent uptake. In the intracellular experiments, each SERS mapping pixel were created through the intensity of the band at $1333 \mathrm{~cm}^{-1}$. (K) Average SERS spectra detected from HeLa cells with different treatments $(n=10)$. (L) Average SERS intensities of HeLa cells under different treatments. The error bars represent the standard deviation of 10 measurements.

Amr: Amiloride; Cpz: Chlorpromazine; Nys: Nystatin; SERS: Surface-enhanced Raman scattering.

suggested that the cell entry for hybrid exosomes was strongly dependent on the clathrin-mediated endocytosis. Furthermore, SERS intensity detected from the cells was observed to be slightly weakened in the presence of amiloride, which indicated that micropinocytosis was less involved (Figure $4 \mathrm{G} \& \mathrm{H}$ ). Similarly, for the cells pretreated with nystatin, the cellular uptake efficiency was found slightly diminished (Figure 4I \& J), which suggested that caveolae-mediated endocytosis rarely participated in the internalization of hybrid exosomes. The average spectra and intensities of HeLa cells with different pretreatments were showed in the Figure $4 \mathrm{~K} \& \mathrm{~L}$, which more clearly demonstrated that the cellular uptake of hybrid exosomes was mainly through the clathrin-mediated and energy-dependent endocytosis. Hybrid exosomes were also stained with the fluorescent dye (CM-DiI) and subject to FACS measurement. As shown in Supplementary Figure 5, a significant reduction of cellular uptake of hybrid exosomes was observed in HeLa cells pretreated at $4^{\circ} \mathrm{C}$ or with chlorpromazine. While amiloride and nystatin treatment only slightly decreased the uptake, the result was consistent with the SERS-based cellular uptake study.

Intracellular trafficking of hybrid exosomes \& the effect of gold nanoparticles in the hybrid After the elucidation of the cellular entry way of SERS-active hybrid exosomes, the intracellular fate of hybrid exosomes is a major concern. It has been reported that the clathrin-mediated endocytosis of nanoparticles often involves a lysosomal pathway inside the cells [38]. To monitor the intracellular trafficking of hybrid exosomes after the clathrin-mediated uptake, the lyso-tracker probes were used to selectively label the acidic subcellular lysosomes. SERS signals were collected to monitor the location of hybrid exosomes while fluorescence signals were used to track the lysosomes. The colocalization experiments were then performed by analyzing the spatial association 

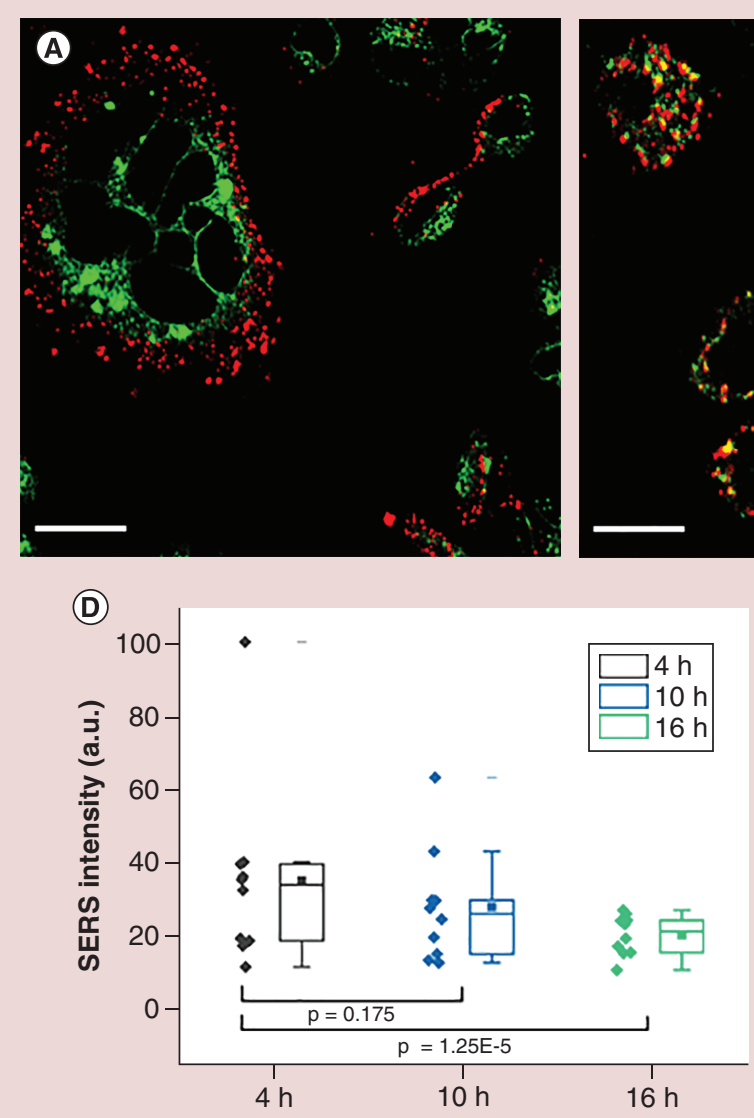
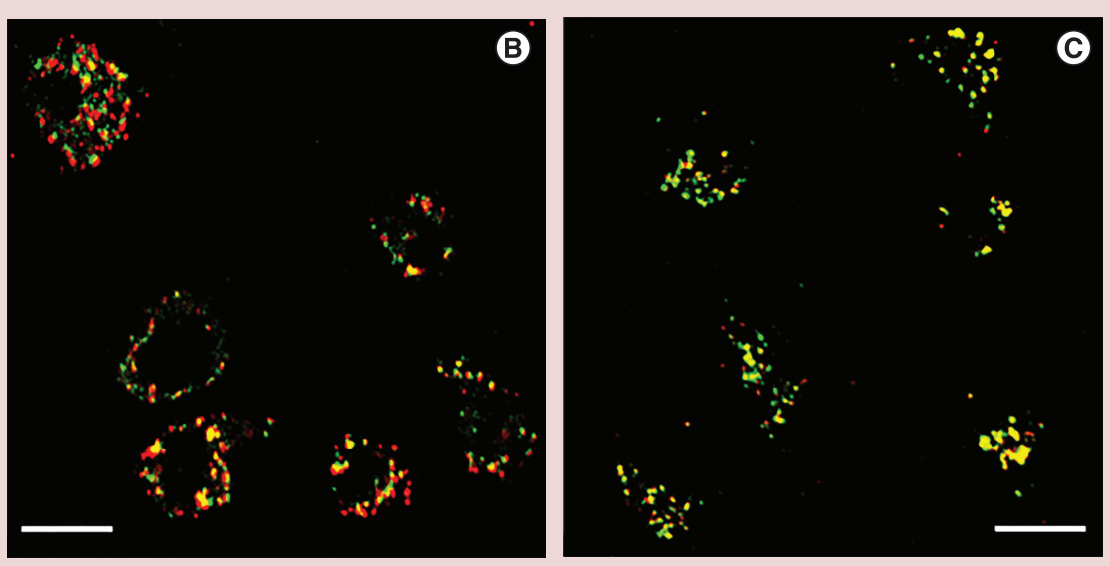

(E)

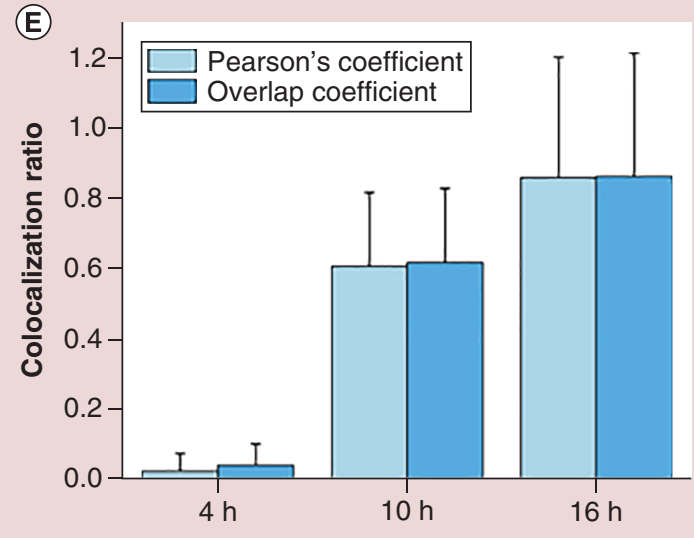

Figure 5. Intracellular tracking of hybrid exosomes. Study on the intracellular distribution of hybrid exosomes with different incubation durations of $4 \mathrm{~h} \mathrm{(A),10} \mathrm{h} \mathrm{(B)} \mathrm{and} 16 \mathrm{~h}$ (C). SERS imaging (red) of hybrid exosomes were obtained by collecting Raman scattering light from 585 to $615 \mathrm{~nm}$; fluorescence imaging (green) of lysosomal compartment were obtained by collection emission light from 505 to $535 \mathrm{~nm}$. (D) Average SERS intensity of per single cell incubated with the hybrid exosomes for 4, 10 and $16 \mathrm{~h}$. (E) Colocalization coefficients between hybrid exosomes and lysosomes. Error bars indicate the standard deviation of coefficients from ten random regions. Scale bar is $10 \mu \mathrm{m}$. SERS: Surface-enhanced Raman scattering.

between hybrid exosomes and lysosomes using the Pearson's correlation coefficient [39] and overlap coefficient [40] (see Supplementary material for a detailed description), which can provide a valuable insight into the intracellular localization of hybrid exosomes. As shown in Figure 5A, less overlapping between SERS and fluorescence punctuates was observed, which exhibited that most hybrid exosomes were not localized in lysosomes at $4 \mathrm{~h}$. As the incubation time prolonged, the colocalization between hybrid exosomes and lysosomes were continuously enhanced (Figure 5B $\& \mathrm{C})$. After $10 \mathrm{~h}$ of incubation, significantly strong colocalization between hybrid exosomes and lysosomes was observed from the merged images. The quantitative colocalization associations measured by Pearson's correlation coefficient and overlap coefficient for different incubation time were shown in Figure 5E, which further confirmed the specific localization of hybrid exosomes within the lysosomes. During the whole culture time, the intracellular SERS intensities were found to be slightly reduced (Figure 5D).

Although the uptake mechanism and intracellular trafficking of hybrid exosomes have been visualized by using SERS, the effect of Au nanoparticles in the hybrid is still lacking, which is definitely vital for the reasonable design of exosome-based nanocarriers. To understand how Au nanoparticles in the hybrids affect the intracellular behaviors of exosomes, the intracellular distribution of pure exosomes was investigated. Experimentally, exosomes were labelled with the lipophilic dye CM-DiI, which were then tracked by fluorescence signals. The colocalization of the exosomes and lysosomes was also analyzed and the results were shown in Figure 6. As the incubation time prolonged, exosomes were observed to be transported to the lysosomes (Figure 6A-C) and the colocalization ratio was correspondingly increased (Figure 6E). The observation of exosomes accumulated in the lysosomal compartment was consistent with 


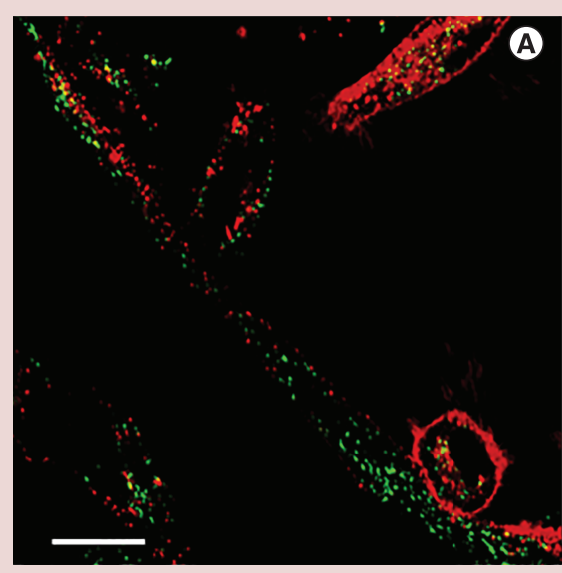

(D)

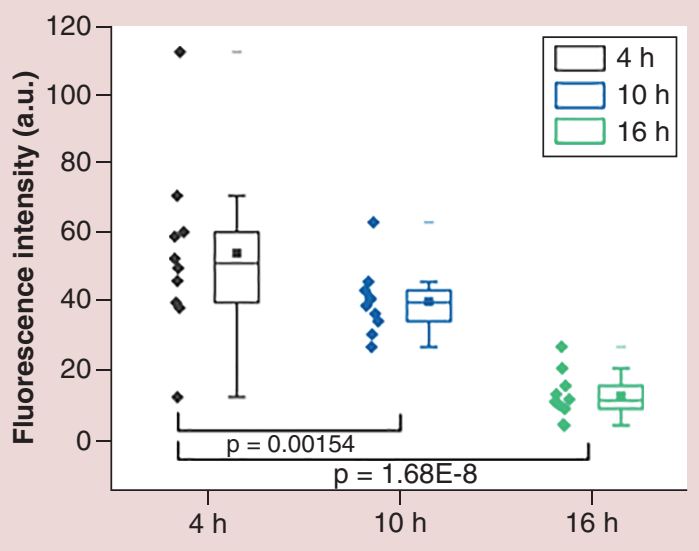

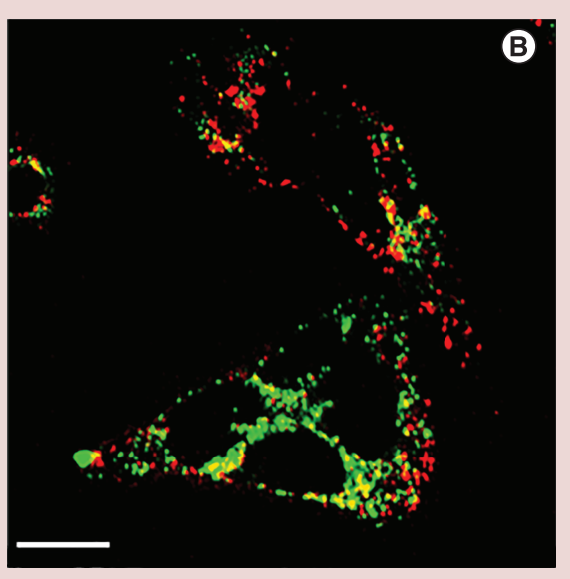

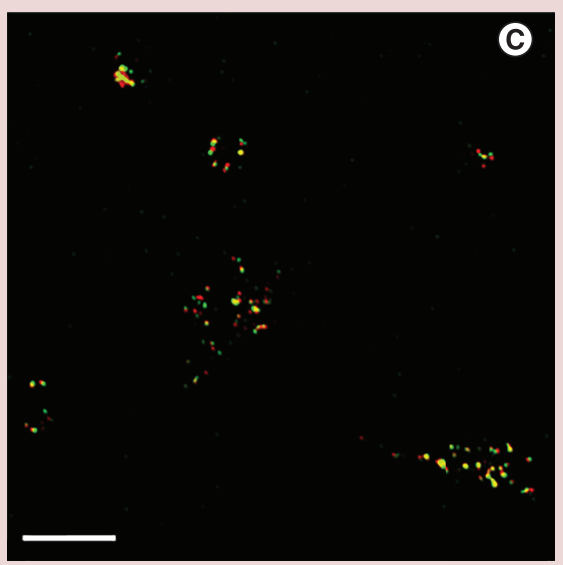

(E)

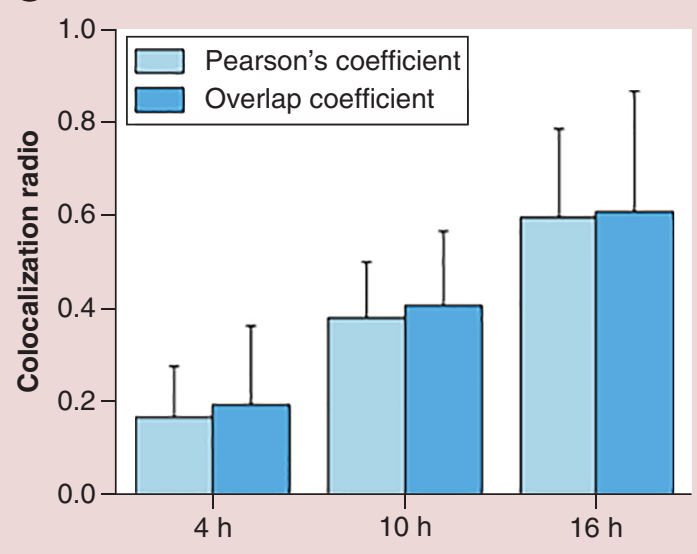

Figure 6. Intracellular tracking of exosomes. Study on the intracellular distribution of CM-Dil-labeled exosomes with different incubation durations of $4 \mathrm{~h}(\mathrm{~A}), 10 \mathrm{~h}$ (B) and $16 \mathrm{~h}$ (C). Fluorescence imaging (red) of CM-Dil-labeled exosomes were obtained by collecting emission light from 685 to $715 \mathrm{~nm}$; fluorescence imaging (green) of lysosomal compartment were obtained by collection emission light from 505 to $535 \mathrm{~nm}$. (D) Average surface-enhanced Raman scattering intensity of per single cell incubated with CM-Dil-labeled exosomes for 4, 10 and 16 h. (E) Colocalization coefficients between CM-Dil-labeled exosomes and lysosomes. Error bars indicate the standard deviation of coefficients from ten random regions. Scale bar is $10 \mu \mathrm{m}$.

previous literatures [41,42]. Moreover, intracellular fluorescence intensities of exosomes were decreased obviously over time, indicating the possible degradation within the lysosomes (Figure 6D). Compared to pure exosomes, hybrid exosomes (Figure 5D) showed a relatively slow cellular degradation rate. Thus, hybrid exosomes had advantages in extending the retention time inside the living cells. Although the retention time varied, the intracellular behaviors of hybrid exosomes are characteristically similar to that of exosomes. Hence, we come to a conclusion that metal nanoparticles have little influence on the intracellular trafficking of exosomes.

As additional evidence, intracellular behaviors of gold nanoprobes were also visualized. As shown in Supplementary Figure 6, the intracellular trafficking of gold nanoprobes showed analogous characteristics as hybrid exosomes. Gold nanoprobes were observed to exhibit strong intracellular retention effect as hybrid exosomes through the time-dependent cellular intensities (Supplementary Figure 6D \& Figure 5D). It is speculated that gold nanoprobes are responsible for the enhanced retention effect of hybrids compared with pure exosomes. Actually, despite the degradation rates varied, metal nanoparticles in the hybrids have little effect on the intracellular behaviors of exosomes.

\section{Discussion}

Exosomes are natural nanovesicles derived by numerous cell types. As the extracellular entities, they are capable of communication with neighboring or distant cells and tissues. Recently, the development of exosome-based 
drug nanocarriers has been of special interest for tumor therapy. Comprehensive research makes the effective labeling strategy as an urgent priority for observing exosomes at the visually cellular or subcellular levels. In our work, a novel kind of exosome/gold nanohybrids is constructed. Au nanoparticles were first prepared and tagged with DTNB molecules and followed by the modification of PAH polymer to form Au nanoprobes. By using glutaraldehyde as bridging linkers, gold nanoprobes were covalently conjugated to exosomes. Exosomes integrated with SERS optical property were denoted as SERS-active hybrid exosomes. TEM images confirmed the successful formation of hybrid exosomes. It is worth noting that exosomes were reported to uptake gold nanoparticles through an active and energy-dependent mechanism [19]. However, this presented exosome/gold was not affected by the incubation temperature whether it was at room temperature or $4^{\circ} \mathrm{C}$. Hence, gold nanoprobes were conjugated to the exosome membrane through the interaction between glutaraldehyde and amino. The dynamic light scattering (DLS) measurement and MTT test indicated that hybrid exosomes had excellent stability and biocompatibility, which were favorable for being used as drug delivery vectors. Intracellular hybrid exosomes were then monitored by SERS signals. It is interesting to note that the SERS intensity dramatically varied at different spots inside the cell, which implied that the distribution of hybrid exosomes was inhomogeneous. This observation highlights the feasibility and good spatial resolution of the SERS technique for tracking exosomes.

By using the SERS technique, cellular uptake of hybrid exosomes by HeLa cells was demonstrated to occur through the energy-dependent and clathrin-mediated endocytosis. After the probing of the cellular entry ways of SERS-active hybrid exosomes, the intracellular fate of hybrid exosomes drew the attention. Through SERSfluorescence joint spectroscopy, the sensing of intracellular trafficking of hybrid exosomes was realized, which indicated that hybrid exosomes tended to adhere onto the cell surface at first and enter the cell through clathrinmediated endocytosis subsequently. After the cellular entry by endocytosis, hybrid exosomes were transported to the lysosomal compartment for degradation. The result was consistent with the specific route of clathrin-associated endocytosis, in which nanomaterials are mostly internalized through clathrin-coated pits and ultimately transported to the lysosomes for degradation [43-45].

Compared to pure exosomes, metal nanoparticles in the hybrids were demonstrated to have little effect on the properties of exosomes while serving as SERS generators. Hence, our novel method of labeling exosomes allows for visualized tracking without compromising functionality. The hybrids can exhibit the optical properties of metallic nanoparticles and simultaneously maintain the intracellular behaviors of exosomes. This mechanistic insight into the role of metal nanoparticles in the hybrid highlights the feasibility of designing programmable exosome/metal hybrid nanoparticles. We reasonably suggest that exosomes labeled with other metal nanoparticles such as silver or gold/silver alloy nanoparticles follow the same principle, which is practical in improving the utility of exosomal nanohybrids as multifunctional nanocarriers.

\section{Conclusion}

In this work, we have demonstrated the successful synthesis of exosome/metal nanohybrids, leading to novel vectors with excellent SERS optical properties. This SERS-active hybrid exosome allowed for the sensing of intracellular trafficking of exosomes through the SERS technique. The results suggested that hybrid exosomes were able to enter into the cells and maintain the optical properties meanwhile. The hybrid exosomes were internalized mainly through clathrin-mediated endocytosis and showed an underlying trend from the cell surface attachment to lysosomal transport. More importantly, metal nanoparticles in the hybrids were demonstrated to have little effect on the intracellular behaviors of exosomes while serving as SERS generators. The results may facilitate the developments of exosome-based drug nanocarriers with clathrin targeting for efficient and personalized therapeutic. Additionally, this valuable insight into the role of metal nanoparticles in the hybrid is significant for breaking down the barriers in designing programmable multifunctional nanocarriers, which will expand the utility of exosomes, ranging from intracellular sensing to drug delivery.

\section{Future perspective}

Exosomes are endogenous vesicles which are currently being used as nanocarriers for drug or gene delivery. Through the combination of inorganic nanoparticles, exosomal nanohybrids show promise as a valuable alternative. The structure and composition of nanohybrids are highly tunable, which can be designed to fit specific requirements for different applications. Specifically, metal-based exosomal nanohybrids can be detected via SERS, rendering them an ideal nanocarriers for theranostics. The spectral diversity and flexible surface modification of these hybrid exosomes also give promise for in vivo imaging at the molecular level in the future. In addition, metal nanoparticles in the 
nanohybrid with suitable surface plasmon resonance adsorption can achieve synergetic efficacy of chemotherapy and photothermal therapy under near-infrared (NIR) light irradiation. The conjugated nanoparticles can also be utilized to realize remote triggered drug release. Hence, these exosomal nanohybrid-based versatile drug-delivery systems offer tremendous opportunities to promote the exosome-based therapeutics forward into more advanced stages in the next few years.

\section{Summary points}

- Facile construction of surface-enhanced Raman scattering-active exosome/metal nanohybrids.

- Optical sensing of intracellular uptake processes of hybrid exosomes using surface-enhanced Raman scattering technique.

- Insight into the influence of metal nanoparticles in the nanohybrids on intracellular behaviors.

- Hybrid exosomes allowing for the visualized sensing of exosomes without compromising their functionality.

- Removing the barriers in programmable design of exosome/metal nanohybrids.

- Providing an important precondition for the utility of hybrid exosomes.

- The promising use of hybrid exosomes for clathrin-targeted drug nanocarriers.

- The potential of hybrid exosomes for combined chemotherapy and photothermal therapy.

Supplementary data

To view the supplementary data that accompany this paper please visit the journal website at: www.futuremedicine.com/doi/sup $\mathrm{pl} / 10.2217 / \mathrm{nnm}-2019-0419$

Author contributions

H Chen and P Ma carried out the design and experiments of this study. C Luo, M Yang and J Li performed the data analysis. H Chen performed the writing of manuscript. X Zhang provided the laboratory instruments for this study. All authors read and approved the final manuscript.

Financial \& competing interests disclosure

This work was supported by National Natural Science Foundation of China (NSFC) (no. 61805143), Shanghai Sailing Program (no. 19YF1435400) and Shanghai Pujiang Program (no. 18PJ1408700). The authors have no other relevant affiliations or financial involvement with any organization or entity with a financial interest in or financial conflict with the subject matter or materials discussed in the manuscript apart from those disclosed.

No writing assistance was utilized in the production of this manuscript.

\section{Open access}

This work is licensed under the Attribution-NonCommercial-NoDerivatives 4.0 Unported License. To view a copy of this license, visit http://creativecommons.org/licenses/by-nc-nd/4.0/

\section{References}

Papers of special note have been highlighted as: $\bullet$ of interest; $\bullet \bullet$ of considerable interest

1. Raposo G, Stoorvogel W. Extracellular vesicles: exosomes, microvesicles, and friends. J. Cell Biol. 200(4), 373-383 (2013).

- Introduces exosomes.

2. Mathivanan S, Ji H, Simpson RJ. Exosomes: extracellular organelles important in intercellular communication. J. Proteomics 73(10), 1907-1920 (2010).

3. Hessvik NP, Llorente A. Current knowledge on exosome biogenesis and release. Cell. Mol. Life Sci. 75(2), 193-208 (2018).

4. Azmi AS, Bao B, Sarkar FH. Exosomes in cancer development, metastasis, and drug resistance: a comprehensive review. Cancer Metastasis Rev. 32(3-4), 623-642 (2013).

5. Sagar G, Sah RP, Javeed N et al. Pathogenesis of pancreatic cancer exosome-induced lipolysis in adipose tissue. Gut 65(7), 1165-1174 (2016).

6. Thery C, Zitvogel L, Amigorena S. Exosomes: composition, biogenesis and function. Nat. Rev. Immunol. 2(8), 569-579 (2002).

- Demonstrates the origination and function of exosomes.

7. Barile L, Vassalli G. Exosomes: therapy delivery tools and biomarkers of diseases. Pharmacol. Ther. 174, 63-78 (2017). 
8. Ferguson SW, Nguyen J. Exosomes as therapeutics: the implications of molecular composition and exosomal heterogeneity. J. Control. Rel. 228, 179-190 (2016).

9. Haney MJ, Klyachko NL, Zhaoa Y et al. Exosomes as drug delivery vehicles for Parkinson's disease therapy. J. Control. Rel. 207, 18-30 (2015).

10. Kamerkar S, Lebleu VS, Sugimoto $\mathrm{H}$ et al. Exosomes facilitate therapeutic targeting of oncogenic KRAS in pancreatic cancer. Nature 546(7659), 498-503 (2017).

11. Din FU, Aman W, Ullah I et al. Effective use of nanocarriers as drug delivery systems for the treatment of selected tumors. Int. J. Nanomed. 12, 7291-7309 (2017).

12. Srivastava A, Amreddy N, Razaq M et al. Exosomes as theranostics for lung cancer. Adv. Cancer Res. 139, 1-33 (2018).

13. Wiklander OPB, Nordin JZ, O'Loughlin A et al. Extracellular vesicle in vivo biodistribution is determined by cell source, route of administration and targeting. J. Extracell. Vesicles 4, 26316-26316 (2015).

14. Verma A, Stellacci F. Effect of surface properties on nanoparticle-cell interactions. Small 6(1), 12-21 (2010).

15. Nakase I, Kobayashi NB, Takatani-Nakase T, Yoshida T. Active macropinocytosis induction by stimulation of epidermal growth factor receptor and oncogenic Ras expression potentiates cellular uptake efficacy of exosomes. Sci. Rep. 5, 10300-10300 (2015).

16. Carnell-Morris P, Tannetta D, Siupa A, Hole P, Dragovic R. Analysis of extracellular vesicles using fluorescence nanoparticle tracking analysis. Methods Mol. Biol. 1660, 153-173 (2017).

- Provides the current analysis of exosomes using fluorescence technique.

17. Zong S, Zong J, Chen C et al. Single molecule localization imaging of exosomes using blinking silicon quantum dots. Nanotechnology 29(6), 065705 (2018)

18. Busato A, Bonafede R, Bontempi P et al. Magnetic resonance imaging of ultrasmall superparamagnetic iron oxide-labeled exosomes from stem cells: a new method to obtain labeled exosomes. Int. J. Nanomed. 11, 2481-2490 (2016).

19. Betzer O, Perets N, Angel A et al. In vivo neuroimaging of exosomes using gold nanoparticles. ACS Nano 11(11), 10883-10893 (2017).

20. Hwang DW, Choi H, Jang SC et al. Noninvasive imaging of radiolabeled exosome-mimetic nanovesicle using (99m)Tc-HMPAO. Sci. Rep. 5, 15636-15636 (2015).

21. Bodelon G, Montes-Garcia V, Fernandez-Lopez C, Pastoriza-Santos I, Perez-Juste J, Liz-Marzan LM. Au@pNIPAM SERRS tags for multiplex immunophenotyping cellular receptors and imaging tumor cells. Small 11(33), 4149-4157 (2015).

- Demonstrates the superiority of surface-enhanced Raman scattering in multiplexing.

22. Yang J, Cui Y, Zong S, Zhang R, Song C, Wang Z. Tracking multiplex drugs and their dynamics in living cells using the label-free surface-enhanced Raman scattering technique. Mol. Pharm. 9(4), 842-849 (2012).

23. Ando J, Fujita K, Smith NI, Kawata S. Dynamic SERS imaging of cellular transport pathways with endocytosed gold nanoparticles. Nano Lett. 11(12), 5344-5348 (2011).

-. Demonstrates the superiority of surface-enhanced Raman scattering in intracellular dynamic tracking.

24. Hu Y, Cheng H, Zhao X et al. Surface-enhanced Raman scattering active gold nanoparticles with enzyme-mimicking activities for measuring glucose and lactate in living tissues. ACS Nano 11(6), 5558-5566 (2017).

25. Frens G. Controlled nucleation for the regulation of the particle size in monodisperse gold suspensions. Nat. Phys. Sci. 241(105), 20-22 (1973).

26. Kelly KL, Coronado E, Zhao LL, Schatz GC. The optical properties of metal nanoparticles: the influence of size, shape, and dielectric environment. J. Phys. Chem. B 107(3), 668-677 (2003).

27. Tian T, Zhang HX, He CP et al. Surface functionalized exosomes as targeted drug delivery vehicles for cerebral ischemia therapy. Biomaterials 150, 137-149 (2018).

28. Maroto R, Zhao Y, Jamaluddin M et al. Effects of storage temperature on airway exosome integrity for diagnostic and functional analyses. J. Extracell. Vesicles 6(1), 1359478-1359478 (2017).

29. Wang Z, Zong S, Chen H, Wu H, Cui Y. Silica coated gold nanoaggregates prepared by reverse microemulsion method: dual mode probes for multiplex immunoassay using SERS and fluorescence. Talanta 86, 170-177 (2011).

30. Grubisha DS, Lipert RJ, Park HY, Driskell J, Porter MD. Femtomolar detection of prostate-specific antigen: an immunoassay based on surface-enhanced Raman scattering and immunogold labels. Anal. Chem. 75(21), 5936-5943 (2003).

31. Oh N, Park JH. Endocytosis and exocytosis of nanoparticles in mammalian cells. Int. J. Nanomed. 9, 51-63 (2014).

32. Canton I, Battaglia G. Endocytosis at the nanoscale. Chem. Soc. Rev. 41(7), 2718-2739 (2012).

33. Zhu D, Wang Z, Zong $S$ et al. Investigating the intracellular behaviors of liposomal nanohybrids via SERS: insights into the influence of metal nanoparticles. Theranostics 8(4), 941-954 (2018).

-. Demonstrates that the nanohybrids preserve the liposomal characteristics.

34. Huang J, Zong C, Shen $\mathrm{H}$ et al. Mechanism of cellular uptake of graphene oxide studied by surface-enhanced Raman spectroscopy. Small 8(16), 2577-2584 (2012). 
35. Rejman J, Bragonzi A, Conese M. Role of clathrin- and caveolae-mediated endocytosis in gene transfer mediated by lipo- and polyplexes. Mol. Ther. 12(3), 468-474 (2005).

36. Koivusalo M, Welch C, Hayashi $\mathrm{H}$ et al. Amiloride inhibits macropinocytosis by lowering submembranous $\mathrm{pH}$ and preventing Rac1 and Cdc42 signaling. J. Cell Biol. 188(4), 547-563 (2010).

37. Huang J, Zong C, Shen $\mathrm{H}$ et al. Mechanism of cellular uptake of graphene oxide studied by surface-enhanced Raman spectroscopy. Small 8(16), 2577-2584 (2012).

38. Sarker SR, Hokama R, Takeoka S. Intracellular delivery of universal proteins using a lysine headgroup containing cationic liposomes: deciphering the uptake mechanism. Mol. Pharm. 11(1), 164-174 (2013).

39. Zinchuk V, Zinchuk O. Quantitative colocalization analysis of confocal fluorescence microscopy images. Curr. Protoc. Cell Biol. 39(1), 4.19.11-14.19.16 (2008).

40. Zinchuk V, Zinchuk O, Okada T. Quantitative colocalization analysis of multicolor confocal immunofluorescence microscopy images: pushing pixels to explore biological phenomena. Acta Histochem. Cytochem. 40(4), 101-111 (2007).

41. Jiang X, Zong S, Chen C, Zhang Y, Wang Z, Cui Y. Gold-carbon dots for the intracellular imaging of cancer-derived exosomes. Nanotechnology 29(17), 175701 (2018).

42. Tian T, Wang Y, Wang H, Zhu Z, Xiao Z. Visualizing of the cellular uptake and intracellular trafficking of exosomes by live-cell microscopy. J. Cell. Biochem. 111(2), 488-496 (2010).

43. Zhai W, He C, Wu L et al. Degradation of hollow mesoporous silica nanoparticles in human umbilical vein endothelial cells. J. Biomed. Mater. Res. Part B 100(5), 1397-1403 (2012).

44. Ma X, Wu Y, Jin $\mathrm{S}$ et al. Gold nanoparticles induce autophagosome accumulation through size-dependent nanoparticle uptake and lysosome impairment. ACS Nano 5(11), 8629-8639 (2011).

- Demonstrates that the lysosomes are responsible for the degradation of nanomaterials.

45. Stern ST, Adiseshaiah PP, Crist RM. Autophagy and lysosomal dysfunction as emerging mechanisms of nanomaterial toxicity. Part. Fibre Toxicol. 9(1), 20 (2012). 